\title{
Enhanced Generic Phase-field Model of Irradiation Materials: Fission Gas Bubble Growth Kinetics in Polycrystalline $\mathrm{UO}_{2}$
}

\section{Nuclear Energy Enabling Technology}

Yulan Li, Shenyang Hu, Robert Montgomery, Fei Gao, Xin Sun Pacific Northwest National Laboratory, Richland, WA 99352

May 2012

FCRD-NEAMS-2012-000134

PNNL-21417 


\section{DISCLAIMER}

This information was prepared as an account of work sponsored by an agency of the U.S. Government. Neither the U.S. Government nor any agency thereof, nor any of their employees, makes any warranty, expressed or implied, or assumes any legal liability or responsibility for the accuracy, completeness, or usefulness, of any information, apparatus, product, or process disclosed, or represents that its use would not infringe privately owned rights. References herein to any specific commercial product, process, or service by trade name, trade mark, manufacturer, or otherwise, does not necessarily constitute or imply its endorsement, recommendation, or favoring by the U.S. Government or any agency thereof. The views and opinions of authors expressed herein do not necessarily state or reflect those of the U.S. Government or any agency thereof. 


\section{Reviewed by:}

National Technical Director, Nuclear Energy

Advanced Modeling and Simulation

\section{Concurred by:}

Acting Director, Advanced Modeling and

Simulation Office

\section{Approved by:}

Deputy Assistant Secretary, Nuclear Energy

John Kelly Date




\title{
Enhanced Generic Phase-field Model of Irradiation Materials: Fission Gas Bubble Growth Kinetics in Polycrystalline $\mathrm{UO}_{2}$
}

\begin{abstract}
Summary
Experiments show that inter-granular and intra-granular gas bubbles have different growth kinetics which results in heterogeneous gas bubble microstructures in irradiated nuclear fuels. A science-based model predicting the heterogeneous microstructure evolution kinetics is desired, which enables one to study the effect of thermodynamic and kinetic properties of the system on gas bubble microstructure evolution kinetics and morphology, improve the understanding of the formation mechanisms of heterogeneous gas bubble microstructure, and provide the microstructure to macroscale approaches to study their impact on thermo-mechanical properties such as thermal conductivity, gas release, volume swelling, and cracking.

In a previous report "Mesoscale Benchmark Demonstration, Problem 1: Mesoscale Simulations of Intragranular Fission Gas Bubbles in $\mathrm{UO}_{2}$ under Post-irradiation Thermal Annealing”[1], a phase-field model was developed to simulate the intra-granular gas bubble evolution in a single crystal during postirradiation thermal annealing. In this work, the phase-field model of intra-granular gas atom and bubble behavior was enhanced by incorporating thermodynamic and kinetic properties at grain boundaries, which can be obtained from atomistic simulations, to simulate fission gas bubble growth kinetics in polycrystalline $\mathrm{UO}_{2}$ fuels. The model takes into account gas atom and vacancy diffusion, vacancy trapping and emission at defects, gas atom absorption and resolution at gas bubbles, internal pressure in gas bubbles, elastic interaction between defects and gas bubbles, and the difference of thermodynamic and kinetic properties in matrix and grain boundaries. The enhanced phase-field model was used to simulate gas atom segregation at grain boundaries and the effect of interfacial energy and gas mobility on gas bubble morphology and growth kinetics in a bi-crystal $\mathrm{UO}_{2}$ during post-irradiation thermal annealing. The preliminary results demonstrate that the model can produce the equilibrium thermodynamic properties and the morphology of gas bubbles at grain boundaries for given grain boundary properties. These predictive capabilities are important because the gas bubble growth and interlinkage depend on the local gas atom segregation, gas bubble morphology and the fluxes of gas atoms and vacancies from the grain. Furthermore, we can use the model to identify the evolution mechanisms behind the inter-granular gas bubble growth kinetics together with atomistic simulations and experiments. More validation of the model capability in polycrystalline is underway.
\end{abstract}

Key words: Phase-field approach; Atomistic simulation; Grain boundary; Fission gas bubbles; Uranium dioxide; Post-irradiation thermal annealing. 


\section{CONTENTS}

FUEL CYCLE R\&D PROGRAM.

ENHANCED GENERIC PHASE-FIELD MODEL OF IRRADIATION MATERIALS: FISSION GAS

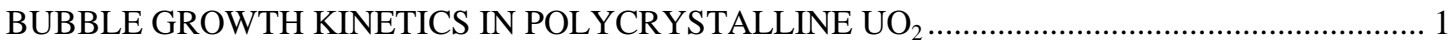

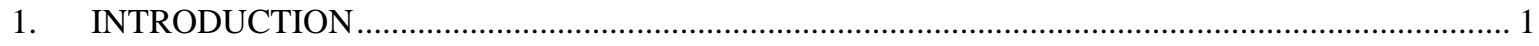

2. GAS BUBBLE MICROSTRUCTURE IN IRRADIATED UO $\mathrm{O}_{2}$ MATERIALS .................................. 3

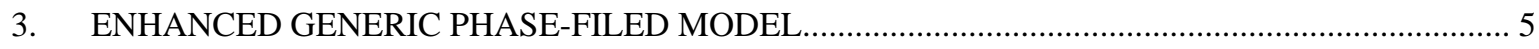

3.1 Definition of microstructure and PF model variables............................................................... 5

3.2 Total free energy .............................................................................................................. 5

3.3 Chemical free energies of the matrix and gas bubble phases ...................................................... 6

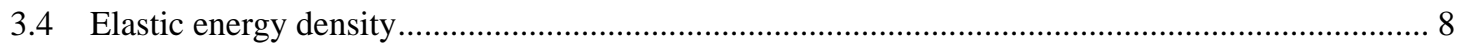

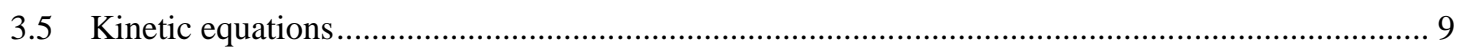

3.6 Diffusivity, mobility, and interfacial energy ......................................................................... 10

3.7 Thermodynamic and kinetic properties at GBs ..................................................................... 10

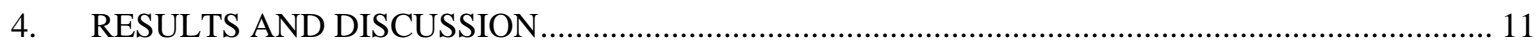

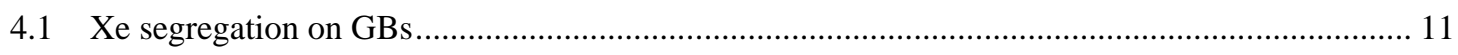

4.2 Effect of interfacial energy and mobility on gas bubble morphologies in a bi-crystal at 2000K .. 12

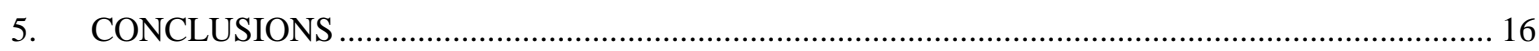

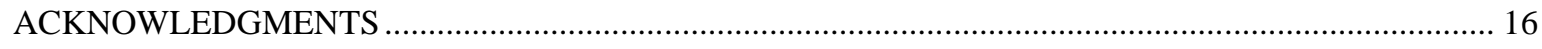

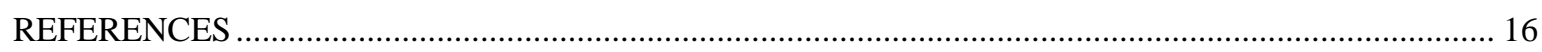

APPENDIX A: THERMODYNAMIC AND KINETIC PROPERTIES OF GRAIN BOUNDARIES............ 18

APPENDIX B: INITIAL CONDITION AND MATERIAL PROPERTIES USED IN SIMULATIONS...... 21

\section{FIGURES}

Figure 1. Schematic process of thermally induced fission gas diffusion and release from fuels..................... 2

Figure 2. Intra-granular bubble behavior from Kashibe et al. [3] ............................................................ 3

Figure 3. Chemical free energies used in our simulations. .......................................................................... 8

Figure 4. (a) Schematic draw of a bi-crystal, (b) temporal evolution of Xe concentration distribution along A-A line shown in (a) for $\Delta E_{X e}^{f}=-0.4 \mathrm{eV}, t^{*}=t / t_{0}, t_{0}=10^{-4} \mathrm{~s}$, and (c) equilibrium Xe

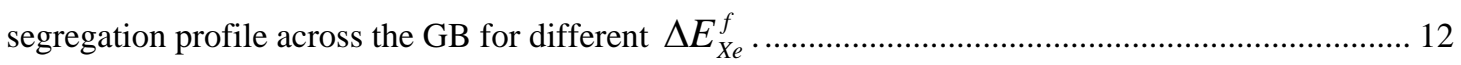

Figure 5. Effect of gas bubble interfacial energies on gas bubble morphology and growth kinetics: (a)

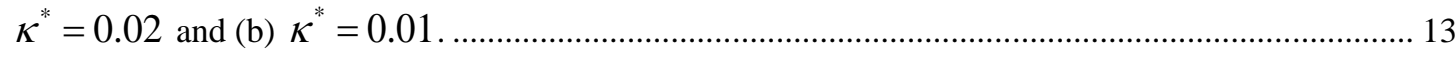

Figure 6. Snapshots of the gas bubble morphology at time $t^{*}=12000$ for $\Delta E_{X e}^{m} / E_{X e}^{m 0}$ being (a) 0 ; (b) 2; (c) 4.

Figure 7. Snapshots of gas bubble temporal evolution from $t^{*}=0$ to $t^{*}=12000$ when $\Delta E_{X e}^{m} / E_{X e}^{m 0}=0$, $\kappa^{*}=0.01$ 15 


\section{ACRONYMS}

${ }^{\circ} \mathrm{C} \quad$ degree(s) Celsius or Centigrade

$\AA^{3} \quad$ cubic Angstrom

$\mathrm{eV} \quad$ electron volt

GPa Giga pascal

GB grain boundary

GWd/MTU gigawatt-days per metric ton uranium

$\mathrm{J} / \mathrm{m}^{2} \quad$ joule per square meter

K Kelvin

$\mathrm{Kr} \quad$ krypton

LWR light water reactor

min minute(s)

MD molecular dynamics

$\mathrm{O} \quad$ oxygen

PF phase-field

s or sec second(s)

U uranium

$\mathrm{UO}_{2} \quad$ uranium dioxide

Xe xenon 


\section{FUEL CYCLE R\&D PROGRAM}

\section{ENHANCED GENERIC PHASE-FIELD MODEL OF IRRADIATION MATERIALS: FISSION GAS BUBBLE GROWTH KINETICS IN POLYCRYSTALLINE UO}

\section{INTRODUCTION}

During operation of light water reactor (LWR), the fission process generates many different fission products, including xenon (Xe) and krypton (Kr) atoms. The noble gas atoms Xe and $\mathrm{Kr}$ represent about $30 \%$ of the fission products produced, and the behavior of the gas atoms is important to the microstructure evolution and overall performance of the nuclear fuel. Because of the extremely low solubility of the noble gas atoms in the $\mathrm{UO}_{2}$ matrix, gas bubbles composed of $\mathrm{Xe}, \mathrm{Kr}$, and other gas atoms precipitate within the $\mathrm{UO}_{2}$ grains and, depending on the temperature conditions, gas atom diffusion will result in nucleation and growth of gas bubbles on the grain boundaries (GBs). Continuum-level mechanisms, such as fission gas release and volumetric swelling due to bubble growth, are included in nuclear fuel performance assessments.

The behavior of fission gas atoms in $\mathrm{UO}_{2}$ has been extensively studied using experiments performed on single crystal and polycrystalline materials under a variety of temperatures and irradiation conditions for more than 50 years [2-8]. From these studies a general picture of the mechanisms that influence the transport and release of fission gas atoms has been developed. Thermally induced fission gas diffusion and release from the fuel generally occur in two stages: 1) diffusion and trapping of single Xe gas atoms within the grains, and 2) formation and inter-linkage of GB bubbles. A schematic of these processes is shown in Figure 1. First, a gas atom is created within a region of radiation-induced vacancies and interstitial atoms caused by the atomic interactions/cascades during the stopping process following fission. Gas atom diffusion assisted by uranium (U) vacancy clusters then takes over until nucleation of small high pressure intra-granular bubbles. These bubbles act as trapping sites for gas atoms until a fission fragment spike causes resolution of Xe atoms back into the matrix. Several transmission electron microscopy studies of irradiated fuel have found that the mean radius of the intra-granular bubbles is approximately $1 \mathrm{~nm}$ with only a slight dependency on the burnup or concentration of the Xe atoms in the matrix $[9,10]$. At sufficient temperatures $\left(>800^{\circ} \mathrm{C}\right)$, gas atoms diffuse to the GBs and nucleate GB bubbles. Some fraction of the gas in the GB bubbles is knocked back into the grain by interactions with fission fragments. The remainder continues to cause the GB bubbles to grow until inter-linkage of bubbles results in venting of the gas out of the fuel. 


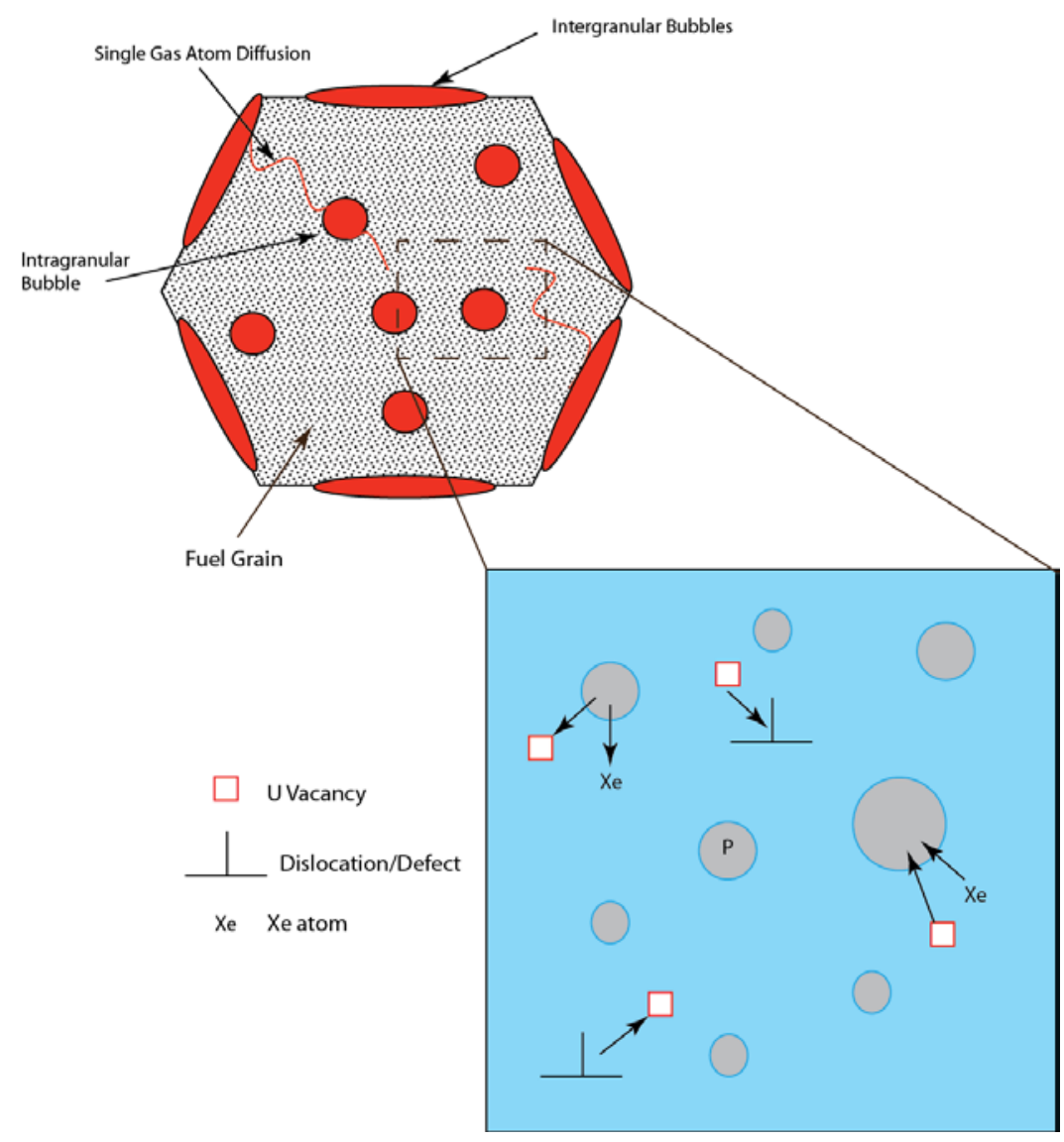

Figure 1. Schematic process of thermally induced fission gas diffusion and release from fuels.

The goal of studying gas atom behavior is to understand the evolution kinetics of the heterogeneous gas bubble microstructure within the grain and along the grain boundaries because it is important to predict gas release, thermal conductivity, fuel swelling, cracking, and fuel performance. Fission gas release is one of the safety issues in nuclear fuel performance. Great effort has been made in developing computational models for the prediction of gas release and fuel performance [11-16]. The most advanced models include FASTGRASS [12], VICTORIA [13], MFPR [14, 15] and FRAPCON[16]. An important feature of these models of fission gas release is the calculation of the gas deposition rate at the boundaries of the fuel grains. In these codes a number of assumptions including the volume average concentration of fission gases in the grain; time and position independent diffusivity; intra-granular gas bubble growth kinetics, and grain growth kinetics are applied in order to empirically calculate the deposition rate. However, it is well known that the gas bubble microstructure within the grain and along the grain boundaries is strongly heterogeneous. Defects such as GBs and dislocations, and the interaction between these defects and vacancies/gas atoms affect the diffusion, absorption and emission of gas atoms and vacancies, gas bubble nucleation and growth kinetics, hence, the gas deposition rate at GBs. Therefore, it is desired to develop a science-based simulation method for studying gas bubble evolution kinetics and assess the effect of defects, thermodynamic and kinetic properties, and different mechanism on gas atom deposition rate at GBs.

The phase-field (PF) approach has been successfully used in predicting 3-D microstructure evolution such as solidification, grain growth, martensitic transition, precipitation, ferroelectric/ferromagnetic transition, 
dislocation dynamics, deformation twin, sintering, and microstructure evolution in irradiated materials [17-23]. The advantages of the PF approach are that 1) no a priori assumption of microstructure morphology is needed; 2) explicitly tracking the interface and topological changes employed in sharp interface methods is not needed; and 3) the continuous description of energy landscape to take into account both short-range and long-range interactions. Application of the PF model has the potential to reduce many of the simplifying assumptions used in engineering scale models to calculate the gas deposition rate at GBs. In the previous report[1], a PF model was developed to simulate the intra-granular gas bubble evolution in a single crystal during post-irradiation thermal annealing. This PF model has now been extended by incorporating thermodynamic and kinetic properties for GB and gas atom interactions, which are obtained from atomistic simulations, to simulate fission gas bubble growth kinetics in polycrystalline $\mathrm{UO}_{2}$ fuels.

\section{GAS BUBBLE MICROSTRUCTURE IN IRRADIATED UO MATERIALS}

Heterogeneous gas bubble microstructure within the grain and along the grain boundaries has been observed in both operating $\mathrm{UO}_{2}$ fuels and post-irradiation thermal annealing $\mathrm{UO}_{2}$ samples [3, 24]. Kashibe et al. [3] investigated the gas bubble evolution in $\mathrm{UO}_{2}$ with a burnup of $23 \mathrm{GWd} / \mathrm{MTU}$ during thermal annealing from $800^{\circ} \mathrm{C}$ to $1800^{\circ} \mathrm{C}$. The pre- and post-thermal annealing appearance of the fission gas bubbles within the grain and along the grain boundaries obtained from transmission electron microscopy are shown in Figure 2. It is clearly seen that the gas bubbles near the GB have much larger size and lower density than those inside the grain. Structural defects such as dislocations and GBs which act as sinks and/or sources of vacancies, interstitials, and gas atoms, play an important role in gas bubble evolution kinetics and the formation of the heterogeneous gas bubble microstructure in polycrystalline $\mathrm{UO}_{2}$ materials. In order to evaluate the role of the different microstructure mechanisms on the intragranular bubble distribution and grain boundary bubble nucleation and growth, the PF model was extended to incorporate the effect of grain boundaries on gas atom diffusion, grain boundary bubble formation, and growth.
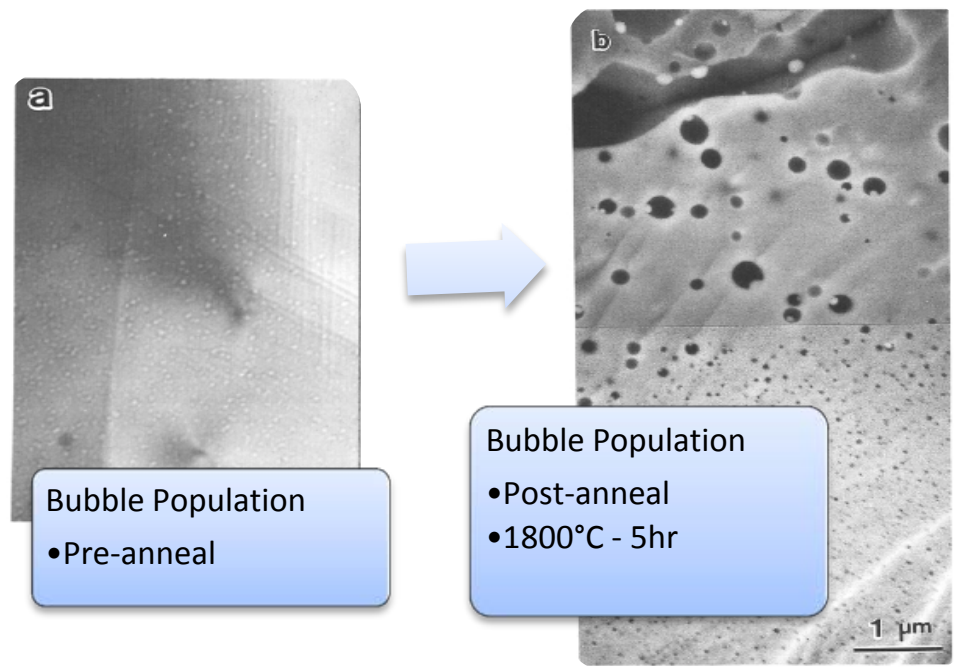

Figure 2. Intra-granular bubble behavior from Kashibe et al. [3]. 
Gas bubble evolution in polycrystalline $\mathrm{UO}_{2}$ materials during post-irradiation thermal annealing is a very complex process. To model the problem, we made the following assumptions:

a) Gas bubble growth needs a continuous supply of U/O vacancies and Xe atoms. Xe atoms may occupy a U vacancy lattice or interstitial lattice depending on valid U vacancy and Xe concentrations. For the simplicity of description, we assume that Xe atoms occupy interstitial lattices. Thus, the $\mathrm{XeU}$ (Xe occupies one $\mathrm{U}$ vacancy) is described by a cluster of a $\mathrm{U}$ vacancy and an interstitial Xe atom. Therefore, a two-sublattice model is used to describe the vacancies and Xe atoms, which allows one to study the effect of vacancy starvation and vacancy emission on gas bubble growth.

b) Xe interstitial, $\mathrm{U}$ vacancy, and the XeU (Xe occupies one U vacancy) have very different mobilities, as reviewed in Appendix A. In addition, other complexes such as XeUO (a Xe occupies one $\mathrm{U}$ and one $\mathrm{O}$ vacancy) and $\mathrm{XeUO}_{2}$ (a Xe occupies one $\mathrm{U}$ vacancy and two $\mathrm{O}$ vacancies) are also mobile and contribute to the diffusion of vacancies and Xe atoms. So the effective mobilities of $\mathrm{U}$ vacancies and Xe atoms are used in the model. In the present simulations the effective migration energy, $3.9 \mathrm{eV}$, for both $\mathrm{U}$ vacancy and Xe atom is used, but different mobilities of $U$ vacancies and Xe atoms can also be used.

c) Gas bubbles formed during low temperature irradiation may be unstable at the higher annealing temperature. So the initial size distribution of gas bubbles may dramatically affect the results of gas bubble size and density evolution. In the simulations, a normal (Gaussian) distribution with a mean radius of $1 \mathrm{~nm}$ and standard deviation of $1 \mathrm{~nm}$ is used to generate the initial gas bubble size distribution.

d) Chemical potential gradient is one driving force for vacancy and Xe diffusion. Kim's model [25] is used to describe the chemical free energy of matrix and gas bubble phases. To efficiently solve the PF evolution equation, we use two parabolic functions to approximate the ideal solution free energy of the matrix with vacancies and Xe atoms and the free energy of the gas bubble phase, which is calculated from the equation of state.

e) Dislocations are sinks or sources of vacancies. Because of the lack of sink and emission strengths, the emission rate of vacancies from dislocations is taken as a model parameter in the simulations.

f) The initial vacancy concentration is assumed as a model parameter since experiments [4] suggested that vacancy emission could be an important mechanism that affects the gas bubble evolution kinetics.

g) O vacancy has a much higher mobility than the U vacancy and Xe atom. But it is not a ratelimiting species in gas bubble growth. Therefore, we neglected $\mathrm{O}$ vacancies. Its effect is accounted for in the effective diffusivity of $U$ vacancies and Xe atoms.

h) The contribution of small gas bubble migration at high temperature to gas bubble evolution is ignored in this model. However, a PF model of void migration can be extended to describe gas bubble migration [23, 26].

i) On GBs, the formation energies of defects such as vacancies and Xe atoms are different from these inside the grains. Therefore, the defect solubility on GBs and inside grains will be different. Spatial dependence of defect formation is assumed in the enhanced PF model. 
j) On GBs, the migration energies of defects such as vacancies and Xe atoms are different from those inside the grains. Spatial dependence of defect mobility is assumed as well.

\section{ENHANCED GENERIC PHASE-FILED MODEL}

\subsection{Definition of microstructure and PF model variables}

In the framework of PF modeling, the microstructure of fission gas bubbles in polycrystalline $\mathrm{UO}_{2}$ can be described by two sets of field variables. One is the concentration variables describing the concentrations of diffusive vacancies and gas atoms. The other one is the order parameter distinguishing grains in the polycrystal and gas bubble phase. In reality, there are a number of mobile vacancies in irradiated $\mathrm{UO}_{2}$ such as single oxygen and uranium vacancies, and small vacancy clusters. Fission reaction also produces a number of fission gas atoms including $\mathrm{Xe}, \mathrm{Kr}$, and He which form the gas phase. For simplicity, we use variable $c_{v}(\mathbf{r}, t)$ to describe the overall vacancy concentration which includes $\mathrm{O}$ and $\mathrm{U}$ vacancies and their vacancy clusters, and $\boldsymbol{c}_{g}(\mathbf{r}, t)$ to describe overall gas atoms $(\mathrm{Xe}, \mathrm{Kr}, \mathrm{He}, \ldots)$ concentration.

Variables $\mathbf{r}$ and $\boldsymbol{t}$ are the spatial coordinate and time, respectively. The vacancies and gas atoms are assumed to diffuse with an effective diffusivity. Thus, two sub lattices, the host lattice and the interstitial lattice, are used to describe the crystal structure. The host lattice is occupied by $\mathrm{U}$ and $\mathrm{O}$ atoms or vacancies while gas atoms occupy the interstitial lattices. The $c_{v}(\mathbf{r}, t)$ and $c_{g}(\mathbf{r}, t)$ are the numbers of moles of vacancies and gas atoms, respectively, in the molar volume of $\mathrm{UO}_{2}$ lattices. Here, we imply that the ratio of $U$ and $O$ vacancies in the molar volume remains $1 / 2$ during absorption, emission and diffusion processes. We assume there are two phases coexisting in the system, i.e., the matrix phase and gas bubble phase. The order parameters are denoted by $\eta_{i}(\mathbf{r}, t),(i=1,2, \cdots, n)$ to distinguish the matrix phase in grain $i$ from others. $n$ is the total number of grains in the simulation cell. Inside of the matrix phase in grain $i, \eta_{i}(\mathbf{r}, t)=1$. Outside of grain $i, \eta_{i}(\mathbf{r}, t)=0$. In the gas bubble phase, all the order parameters $\eta_{i}(\mathbf{r}, t),(i=1,2, \cdots, n)$ are set equal to zero. The equilibrium thermodynamic values of concentrations and order parameters are: $\left(\eta=1, c_{v}=c_{v}^{m, e q}, c_{g}=c_{g}^{m, e q}\right)$ at the matrix phase and $\left(\eta=0, c_{v}=c_{v}^{b, e q}, c_{g}=c_{g}^{b, e q}\right)$ at the gas bubble phase where $\eta=\sum_{i=1}^{n} \eta_{i}$. Order parameter $\eta_{i}(\mathbf{r}, t)$ smoothly changes from 1 to 0 across its corresponding interfaces including GBs and gas bubble interfaces. The vacancy concentration $c_{v}(\mathbf{r}, t)$ and gas atom concentration $c_{g}(\mathbf{r}, t)$ also smoothly vary from ( $\left.c_{v}^{b, e q}, c_{g}^{b, e q}\right)$ to $\left(c_{v}^{m, e q}, c_{g}^{m, e q}\right)$ from the gas bubble phase to the matrix phase.

\subsection{Total free energy}

The total free energy of the system includes the chemical free energy, gradient energy, and long-range interaction energy, and is written as a function of the PF variables as

$$
E=\int_{V}\left[F\left(c_{v}, c_{g}, \eta_{1}, \cdots, \eta_{n}, T\right)+\sum_{i=1}^{n} \sum_{j=1}^{n} \frac{\kappa_{i j}^{2}}{2}\left|\nabla \eta_{i} \nabla \eta_{j}\right|+\frac{\kappa^{2}}{2}\left(\left|\nabla c_{v}\right|^{2}+\left|\nabla c_{g}\right|^{2}\right)+U^{\text {def }}\left(\varepsilon_{i j}\right)\right] d V \text {, }
$$


where $V$ is the system volume, $F\left(c_{v}, c_{g}, \eta_{1}, \cdots, \eta_{n}, T\right)$ is the chemical free energy density, $T$ is absolute temperature, $\kappa_{i j}$ and $\kappa$ are the gradient coefficients associated with interfacial energy of GBs and gas bubbles, $U^{\text {def }}$ is the elastic energy density associated with the lattice mismatch of the defects including distributed gas bubbles, vacancies, gas atoms, and GBs, and $\varepsilon_{i j}(i, j=1,2,3)$ are elastic strain components.

Following Kim's solidification model [25], the chemical free energy density of the system is constructed as

$$
F\left(c_{v}, c_{g}, \eta_{1}, \cdots, \eta_{n}, T\right)=h(\eta) f^{m}\left(c_{v}^{m}, c_{g}^{m}, T\right)+[1-h(\eta)] f^{b}\left(c_{v}^{b}, c_{g}^{b}, T\right)+w g\left(\eta_{1}, \cdots, \eta_{n}\right),
$$

where $\left(f^{m}, f^{b}\right),\left(c_{v}^{m}, c_{g}^{m}\right)$ and $\left(c_{v}^{b}, c_{g}^{b}\right)$ are the chemical free energy densities, vacancy and gas atom concentrations of the matrix and the gas bubble phases, respectively. $g\left(\eta_{1}, \cdots, \eta_{n}\right)$ is a double-well potential for each $\eta_{i}$ and the simplest form is $g\left(\eta_{1}, \cdots, \eta_{n}\right)=\sum_{i=1}^{n}\left[\eta_{i}^{2}\left(1-\eta_{i}\right)^{2}\right]$ and $w$ is the height of the double well. $h(\eta)=3 \eta^{2}-2 \eta^{3}\left(\eta=\sum_{i=1}^{n} \eta_{i}\right)$ is an interpolation function describing the volume fraction of matrix phase. The Kim's model assumes that any point within the gas bubble interface region is considered as a mixture of the matrix and gas bubble phases with the same inter-diffusion potential. Therefore, the concentrations satisfy the following chemical equilibrium and mass balance equations,

$$
\begin{aligned}
& c_{v}=h(\eta) c_{v}^{m}+[1-h(\eta)] c_{v}^{b}, \quad c_{g}=h(\eta) c_{g}^{m}+[1-h(\eta)] c_{g}^{b}, \\
& \frac{\partial f^{m}\left(c_{v}^{m}, c_{g}^{m}, T\right)}{\partial c_{v}^{m}}=\frac{\partial f^{b}\left(c_{v}^{b}, c_{g}^{b}, T\right)}{\partial c_{v}^{b}}, \frac{\partial f^{m}\left(c_{v}^{m}, c_{g}^{m}, T\right)}{\partial c_{g}^{m}}=\frac{\partial f^{b}\left(c_{v}^{b}, c_{g}^{b}, T\right)}{\partial c_{g}^{b}} .
\end{aligned}
$$

From eq. (3), we can obtain $\left(c_{v}^{m}, c_{g}^{m}\right)$ and $\left(c_{v}^{b}, c_{g}^{b}\right)$ for given $\left(c_{v}, c_{g}\right)$.

\subsection{Chemical free energies of the matrix and gas bubble phases}

Using the ideal solution assumption, the chemical free energy density of the matrix phase with vacancy and gas concentrations $C_{v}(\mathbf{r}, t)$ and $c_{g}(\mathbf{r}, t)$ can be written as

$$
\begin{aligned}
f^{m}\left(c_{v}^{m}, c_{g}^{m}, T\right)= & \frac{1}{\Omega}\left\{\Re T\left[c_{v}^{m} \ln \left(c_{v}^{m}\right)+\left(1-c_{v}^{m}\right) \ln \left(1-c_{v}^{m}\right)\right]+N_{A} E_{v}^{f} c_{v}^{m}\right. \\
& \left.+\Re T\left[c_{g}^{m} \ln \left(c_{g}^{m}\right)+\left(1-c_{g}^{m}\right) \ln \left(1-c_{g}^{m}\right)\right]+N_{A} E_{g}^{f} c_{g}^{m}\right\},
\end{aligned}
$$

where $\mathfrak{R}=8.314[\mathrm{~J} /(\mathrm{mol} \cdot \mathrm{K})]$ is the gas constant, $N_{A}=6.022 \times 10^{23}\left[\mathrm{~mol}^{-1}\right]$ is the Avogadro constant. $E_{v}^{f}$ and $E_{g}^{f}$ are the formation energies of a vacancy and a gas atom, respectively. $\Omega$ is the molar volume of $\mathrm{UO}_{2}$ and $\Omega=2.53 \times 10^{-5}\left[\mathrm{~m}^{3} / \mathrm{mol}\right]$.

In simulations, $f^{m}\left(c_{v}^{m}, c_{g}^{m}, T\right)$ is replaced approximately by parabolic functions as 


$$
f^{m}\left(c_{v}^{m}, c_{g}^{m}, T\right)=A_{12}\left(c_{v}^{m}-c_{v}^{m, e q}\right)^{2}+B_{12}\left(c_{g}^{m}-c_{g}^{m, e q}\right)^{2},
$$

where $c_{v}^{m, e q}=\exp \left(-E_{v}^{f} / k_{B} T\right)$ and $c_{g}^{m, e q}=\exp \left(-E_{g}^{f} / k_{B} T\right)$ are the thermal equilibrium solubility of vacancies and gas atoms, respectively. $k_{B}=1.381 \times 10-23[\mathrm{~J} / \mathrm{K}]$ is the Boltzmann constant. Both $f^{m}\left(c_{v}^{m}, c_{g}^{m}, T\right)$ in eqs. (4) and (5) have the same first derivatives at a given concentration $\left(c_{v}^{0}, c_{g}^{0}\right)$.

If assuming a $\mathrm{UO}_{2}$ vacancy has the same formation energy as a Xe atom, $E_{v}^{f}=E_{g}^{f}=E^{f}=3 \mathrm{eV}$. In the following simulations, $\left(c_{v}^{0}, c_{g}^{0}\right)=(0.01,0.01), B_{12}=A_{12}$ and

$$
\begin{aligned}
& \frac{A_{12}}{C_{44}}=\frac{-a_{2}+3 a_{1}}{4\left(c_{v}^{0}-c_{v}^{m, e q}\right)}+\frac{a_{2}-a_{1}}{2400\left(c_{v}^{0}-c_{v}^{m, e q}\right)} T \\
& a_{1}=0.178605-0.0030782 \log \left(1-c_{v}^{0}\right)+0.0030782 \log \left(c_{v}^{0}\right), \\
& a_{2}=0.178605-0.00923461 \log \left(1-c_{v}^{0}\right)+0.00923461 \log \left(c_{v}^{0}\right) .
\end{aligned}
$$

For simplicity, the chemical free energy density of the gas bubble phase is assumed to be parabolic functions of $c_{v}^{b}$ and $c_{g}^{b}$ as

$$
f^{b}\left(c_{v}^{b}, c_{g}^{b}, T\right)=A_{22}\left(c_{v}^{b}\right)^{2}+A_{21} c_{v}^{b}+A_{20}+B_{22}\left(c_{g}^{b}\right)^{2}+B_{21} c_{g}^{b}+B_{20} .
$$

If $c_{v}^{b, e q}$ and $c_{g}^{b, e q}$ are the equilibrium concentrations of the gas bubble phase, $c_{v}^{b, e q}=1$ and $c_{g}^{b, e q}$ can be obtained from the equation of state of Xenon. We used Ronchi's results of Xenon [27] to determine $c_{g}^{b, e q} \approx 0.7$. The left two unknown coefficients (e.g., $A_{22}$ and $B_{22}$ ) will be determined by given ( $\left.c_{v}^{\text {cross }}, c_{v}^{\text {cross }} ; f^{\text {cross }}\right)$, i.e., $f^{m}\left(c_{v}^{\text {cross }}, c_{v}^{\text {cross }}, T\right)=f^{b}\left(c_{v}^{\text {cross }}, c_{v}^{\text {cross }}, T\right)=f^{\text {cross }}$. Therefore, $A_{22}=\frac{A_{12} f^{\text {cross }}}{\left(\sqrt{A_{12}}\left(c_{v}^{m, e q}-c_{v}^{b, e q}\right)+\sqrt{f^{\text {cross }}}\right)^{2}}, \quad B_{22}=\frac{B_{12} f^{\text {cross }}}{\left(\sqrt{B_{12}}\left(c_{g}^{m, e q}-c_{g}^{b, e q}\right)+\sqrt{f^{\text {cross }}}\right)^{2}}$. If $\frac{f^{\text {cross }}}{C_{44}}=0.5$, then the chemical free energy is completely determined as shown in Figure 3. 


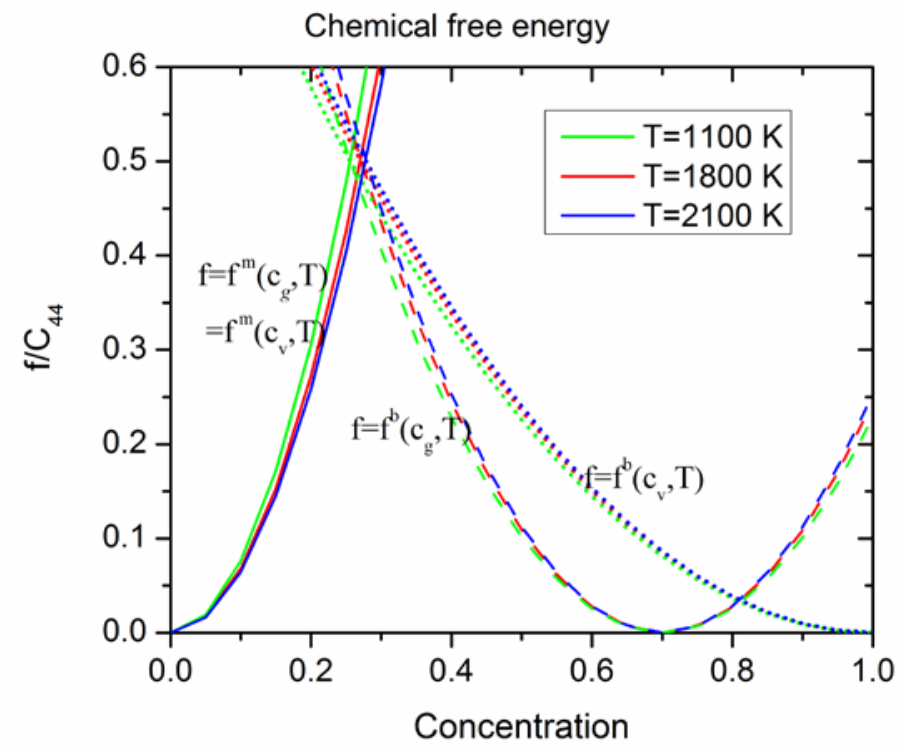

Figure 3. Chemical free energies used in our simulations.

\subsection{Elastic energy density}

The last term of the right side of eq. (1) is the elastic energy. If we assume that the variation of stress-free lattice parameter of the matrix phase, $a$, with the given concentrations of vacancies and gas atoms, obeys the Vegard's law, the local stress-free strain caused by the defect inhomogeneity is given by

$$
\varepsilon_{i j}^{* m}=\left[\varepsilon^{v 0}\left(c_{v}-c_{v}^{m, e q}\right) \delta_{i j}+\varepsilon^{g 0}\left(c_{g}-c_{g}^{m, e q}\right) \delta_{i j}\right] h(\eta),
$$

where $\varepsilon^{v 0}=(1 / a) d a / d c_{v}$ and $\varepsilon^{g 0}=(1 / a) d a / d c_{g}$ are the expansion coefficients of the lattice parameter due to the introduction of vacancies and interstitials, respectively, and $\delta_{i j}$ is the Kroneckerdelta function. The vacancy and gas concentrations are high in the gas bubble phase where the Vegard's law may no long be applicable. The stress-free strain in the gas bubble phase is described as

$$
\varepsilon_{i j}^{* b}(\mathbf{r})=\varepsilon^{b 0}\left(c_{g}, T\right) \delta_{i j}[1-h(\eta)],
$$

where $\varepsilon^{b 0}\left(c_{g}, T\right)$ can be estimated as $\varepsilon^{b 0}\left(c_{g}, T\right)=P /\left(C_{11}+2 C_{12}\right)$ where $\mathrm{P}$ is the pressure inside of the gas bubble phase and can be obtained from the equation of state, and $C_{11}$ and $C_{12}$ are the elastic constants of the gas bubble phase. Thus, the total stress-free strain tensor is

$$
\begin{aligned}
\varepsilon_{i j}^{*} & =\varepsilon_{i j}^{* m}+\varepsilon_{i j}^{* b} \\
& =\left[\varepsilon^{v 0}\left(c_{v}-c_{v}^{m, e q}\right) \delta_{i j}+\varepsilon^{g 0}\left(c_{g}-c_{g}^{m, e q}\right) \delta_{i j}\right] h(\eta)+\varepsilon^{b 0}\left(c_{g}, T\right) \delta_{i j}[1-h(\eta)]
\end{aligned}
$$

The elastic energy density $U^{\text {def }}$ is calculated by:

$$
U^{d e f}=\frac{1}{2} \lambda_{i j k l} \varepsilon_{i j}^{e l} \varepsilon_{k l}^{e l}
$$


where the summation convention over the repeated indexes is used. $\lambda_{i j k l}$ is the elastic constant tensor of the system (its Voigt notation is $C_{i j}$ ). $\varepsilon_{i j}^{e l}$ is the elastic strain which is calculated by

$$
\varepsilon_{i j}^{e l}=\bar{\varepsilon}_{i j}+\delta \varepsilon_{i j}(\mathbf{r})-\varepsilon_{i j}^{*}(\mathbf{r}) \text {, }
$$

where $\bar{\varepsilon}_{i j}$ is the homogeneous strain characterizing the macroscopic shape and volume change, $\delta \varepsilon_{i j}(\mathbf{r})$ is the heterogeneous strain of $\int_{V} \delta \varepsilon_{i j}(\mathbf{r}) d V=0$. For elastic inhomogeneous solids, such as $\mathrm{UO}_{2}$ matrix with voids and gas bubbles, the elastic solution can be obtained using the iteration method [28]. For simplicity, we assume the gas phase has the same elastic constants as $\mathrm{UO}_{2}$ and of cubic anisotropy with $C_{11} / C_{44}=6.17, C_{12} / C_{44}=1.89, C_{44}=64[\mathrm{GPa}]$. [29]

\subsection{Kinetic equations}

In all PF models, the temporal and spatial evolution of the field variables follows the same set of kinetic equations. A conserved field, e.g., concentration field, evolves with time according to the Cahn-Hilliard equation [30], whereas a non-conserved field, e.g., the order parameter field, is governed by the AllenCahn equation [31]. The evolution equations of $c_{v}, c_{g}$, and $\eta_{i}$ are written as

$$
\begin{aligned}
\frac{\partial c_{v}}{\partial t} & =\nabla \cdot\left\{M_{v v} \nabla\left(\frac{\partial\left(F+U^{d e f}\right)}{\partial c_{v}}-\kappa \nabla^{2} c_{v}\right)\right\}+\dot{\omega}\left(\rho_{\text {dis }}, c_{v}, \sigma_{i j}\right), \\
\frac{\partial c_{g}}{\partial t} & =\nabla \cdot\left\{M_{g g} \nabla\left(\frac{\partial\left(F+U^{d e f}\right)}{\delta c_{g}}-\kappa \nabla^{2} c_{g}\right)\right\}, \\
\frac{\partial \eta_{i}}{\partial t} & =-L\left(\frac{\partial F}{\partial \eta_{i}}+\frac{\partial U^{d e f}}{\partial \eta_{i}}-\kappa_{i i}^{2} \nabla^{2} \eta_{i}-\sum_{i \neq j} \kappa_{i j}^{2} \nabla^{2} \eta_{j}\right) \\
= & -L\left[-\kappa_{i i}^{2} \nabla^{2} \eta_{i}-\sum_{i \neq j} \kappa_{i j}^{2} \nabla^{2} \eta_{j}+w g^{\prime}\left(\eta_{i}\right)\right] \\
& -L\left\{h^{\prime}\left(\eta_{i}\right)\left[\left(f^{m}-f^{b}\right)-\left(c_{v}^{m}-c_{v}^{g}\right) \frac{\partial f^{m}}{\partial c_{v}^{m}}+\left(c_{g}^{m}-c_{g}^{g}\right) \frac{\partial f^{m}}{\partial c_{g}^{m}}\right]+\frac{\partial U^{d e f}}{\partial \eta_{i}}\right\},
\end{aligned}
$$

where $M_{v v}$ and $M_{g g}$ represent the mobility of vacancies and gas atoms and are determined from the diffusivity by $M_{v v}=D /\left.\left(\frac{\partial^{2} f^{m}}{\partial c_{v}^{2}}\right)\right|_{c_{v}=c_{v}^{m, e q}}$ and $M_{g g}=D /\left.\left(\frac{\partial^{2} f^{m}}{\partial c_{g}^{2}}\right)\right|_{c_{g}=c_{g}^{m, e q}} . L$ is the interface mobility coefficient. $\dot{\omega}\left(\rho_{d i s}, c_{v}, \sigma_{i j}\right)$ is the vacancy emission rate which depends on dislocations $\rho_{\text {dis }}$, local stress $\sigma_{i j}$, and vacancy concentration $C_{v}(\mathbf{r}, t)$. 


\subsection{Diffusivity, mobility, and interfacial energy}

Assume vacancies and gas atoms have the same diffusivity of $D=0.5 \times 10^{-4} \exp \left[-11604.5 E_{m} / T\right]$ $\left[\mathrm{m}^{2} / \mathrm{s}\right.$ ] with $E_{m}$ being vacancy or gas atom migration energy in $\mathrm{eV}$. The gradient energy coefficients $\kappa_{i i}$, $\kappa_{i j}(i \neq j), \kappa$, and double well height $w$ are model parameters which can be determined by the interfacial energy and interface thickness of the gas bubbles and GBs. The relationship between model parameters and material properties can be derived with the equilibrium solutions [19, 32]. In the present simulations, the model parameters are estimated by the following relations:

$$
\begin{array}{ll}
\gamma_{G B} \propto\left(\kappa_{i i}+\kappa_{i j}\right) \sqrt{w}, & \gamma_{\text {Bubble }} \propto \kappa_{i i} \sqrt{w}+\kappa \sqrt{\Delta f}, \\
2 \lambda_{G B} \propto\left(\kappa_{i i}+\kappa_{i j}\right) / \sqrt{w}, & 2 \lambda_{\text {Bubble }} \propto \frac{\kappa_{i i}}{\sqrt{w}}+\frac{\kappa}{\sqrt{\Delta f}},
\end{array}
$$

where $\gamma_{G B}$ and $\gamma_{\text {Bubble }}$ are the interfacial energies of the GB and gas bubble interface, respectively. $2 \lambda_{G B}$ and $2 \lambda_{\text {Bubble }}$ are the thickness of the GB and gas bubble interface, respectively. And $\Delta f$ is the height of the double well of free energy $h(\eta) f^{m}\left(c_{v}^{m}, c_{g}^{m}, T\right)+[1-h(\eta)] f^{b}\left(c_{v}^{b}, c_{g}^{b}, T\right)$.

\subsection{Thermodynamic and kinetic properties at GBs}

Like in single crystal, the thermodynamic and kinetic properties of defects near GBs can be calculated by atomistic simulations. The current model aims to incorporate the thermodynamic and kinetic properties of gas bubble evolution in polycrystalline $\mathrm{UO}_{2}$ materials. To do so, spatial dependences of formation energies and mobility of defects are described by:

$$
\begin{aligned}
& E_{v, X e}^{f}=E_{v, X e}^{f 0}+\Delta E_{v, X e}^{f} y_{v, X e}^{f}\left(\mathbf{r},\left|\nabla \eta_{i} \nabla \eta_{j}\right|_{i \neq j}\right) \\
& E_{v, X e}^{m}=E_{v, X e}^{m 0}+\Delta E_{v, X e}^{m} y_{v, X e}^{m}\left(\mathbf{r},\left|\nabla \eta_{i} \nabla \eta_{j}\right|_{i \neq j}\right)
\end{aligned}
$$

where $E_{v, X e}^{f 0}$ is the formation energy of vacancy/Xe inside the grain, $\Delta E_{v, X e}^{f}$ is the difference of the formation energies at the GB and inside of the grain, $y_{v, X e}^{f}\left(\mathbf{r},\left|\nabla \eta_{i} \nabla \eta_{j}\right|_{i \neq j}\right)$ is the spatial dependent function of formation energy. $E_{v, X e}^{m 0}$ is the migration energy of vacancy/Xe inside the grain, $\Delta E_{v, X e}^{m}$ is the difference of the migration energies at the GB and inside of the grain, $y_{v, X e}^{m}\left(\mathbf{r},\left|\nabla \eta_{i} \nabla \eta_{j}\right|_{i \neq j}\right)$ is the spatial dependent function of the migration energy. Generally speaking, the spatial dependence of vacancy/Xe formation energy and migration energy can be fitted from atomistic simulation results. The related atomistic simulation methods are described in details in Appendix A. 


\section{RESULTS AND DISCUSSION}

To validate the developed PF model, we examined the gas atom segregation at GBs, the effect of interfacial energy and mobility on inter-granular gas bubble morphology and gas bubble growth kinetics in a bi-crystal using a 2-D computational model. A simulation cell $512 \mathrm{dx} \times 1 \mathrm{dy} \times 64 \mathrm{dz}$, and grad size $\mathrm{dx}=\mathrm{dy}=\mathrm{dz}=l_{0}=1 \mathrm{~nm}$ were used. Considering the fact that the size of grains in $\mathrm{UO}_{2}$ pellets is about 10 microns, the simulation cell is 0.512 micron. Therefore, the simulation cell is too small to fully represent the interaction between intra-granular gas bubbles and inter-granular gas bubbles. In this work, we focus on examining the thermodynamic properties at GBs. In the simulations, the initial conditions, thermodynamic and kinetic properties and model parameters are listed in the Table B - 1 and Table B - 2 of Appendix B which were from a previous report [1]. The migration energies of vacancies and gas atoms are set to be the same as $E_{m}=E_{X e}^{m 0}$. The height of the double wells is $w / C_{44}=0.014$.

\subsection{Xe segregation on GBs}

Since the formation energy of Xe/vacancy at GBs is different from that inside of grains, its solubility is different inside of grains and at GBs, hence, Xe/vacancy segregation or depletion at GBs occurs. To examine Xe segregation at GBs we consider a bi-crystal without any initial gas bubble presence as shown in Figure 4(a). The initial gas concentrations of vacancies and Xe atoms in the matrix are 0.0042. Figure 4(b) shows the time evolution of Xe concentration distribution across the interface along A-A line as shown in Figure 4(a). The formation energy of Xe atom/vacancy at the GB is $3.5 \mathrm{eV}$ while it is $3.9 \mathrm{eV}$ inside of the grain. It is clearly seen from Figure 4(b) that Xe concentration gradually reaches its equilibrium state with time and its equilibrium concentration at the GB is higher than that inside of the grain due to smaller formation energy at the GB. The dependence of Xe segregation on its formation energy at GBs is plotted in Figure 4(c). 


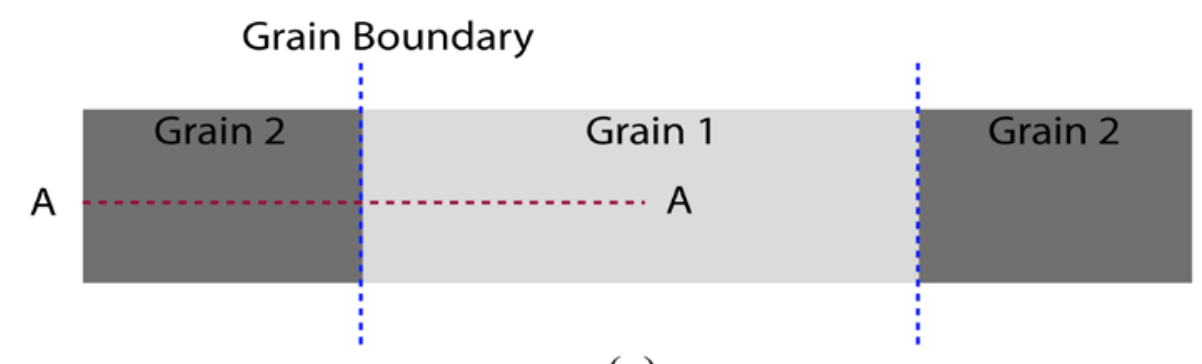

(a)

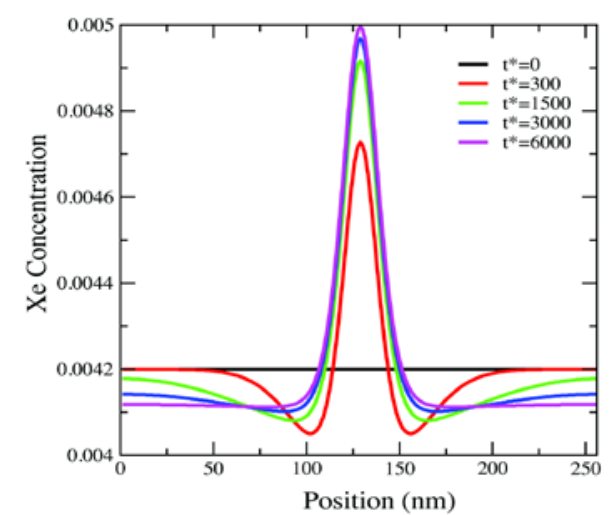

(b)

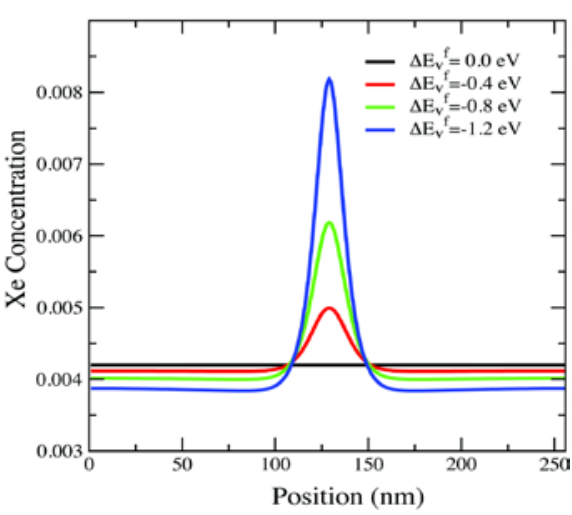

(c)

Figure 4. (a) Schematic draw of a bi-crystal, (b) temporal evolution of Xe concentration distribution along A-A line shown in (a) for $\Delta E_{X e}^{f}=-0.4 \mathrm{eV}, t^{*}=t / t_{0}, t_{0}=10^{-4} \mathrm{~s}$, and (c) equilibrium Xe segregation profile across the GB for different $\Delta E_{X e}^{f}$.

\subsection{Effect of interfacial energy and mobility on gas bubble morphologies in a bi-crystal at $2000 \mathrm{~K}$}

In order to study the effect of interfacial energy and gas atom mobility, we started a simulation with initially random distributed gas bubbles of a density $9 \times 10^{23} / \mathrm{m}^{3}$ and a mean diameter of $2 \mathrm{~nm}$. The GB gradient coefficients of $\kappa_{i i} / \kappa_{0}=0.012$ and $\kappa_{i j} / \kappa_{0}=0.0(i \neq j)$ with $\kappa_{0}=\left(l_{0}^{2} C_{44}\right)$ are used in the simulations. Figure 5 shows the gas bubble evolution in a half of the simulation cell. The color bar denotes Xe concentration. The white line shows the left GB in Figure 4(a). The gas bubbles in Figure 5(a) have a larger interfacial energy (the dimensionless gradient coefficient $\kappa^{*}=\kappa / \kappa_{0}=0.02$ ) than that in Figure 5(b) where $\kappa^{*}=0.01$. As expected, the gas bubbles in Figure 5(a) have a larger contact angle at the GB than that in Figure 5(b). From the GB evolution, we can see that interaction between the GB and nearby gas bubbles results in the GB migration. 


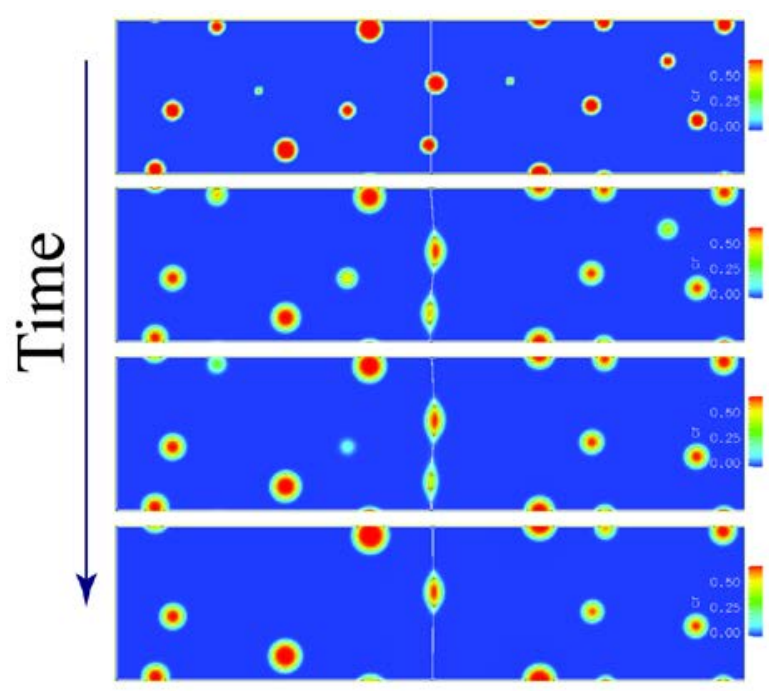

(a)

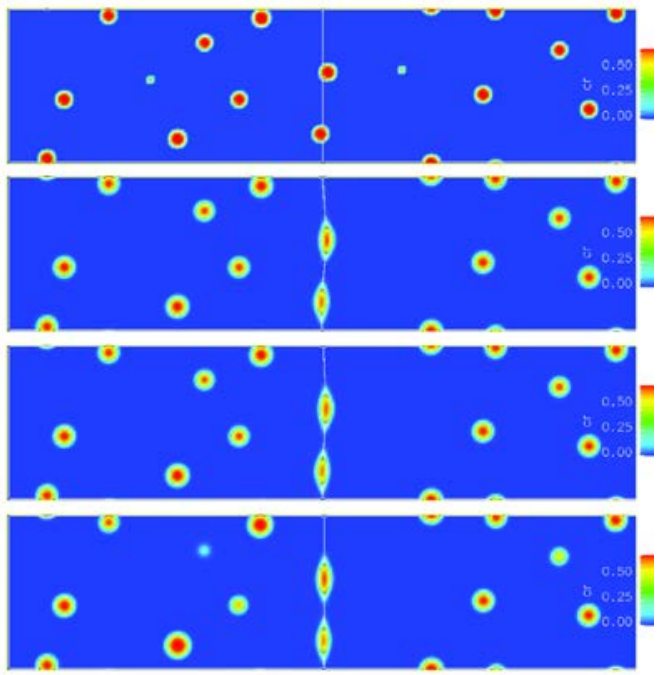

(b)

Figure 5. Effect of gas bubble interfacial energies on gas bubble morphology and growth kinetics: (a)

$$
\kappa^{*}=0.02 \text { and (b) } \kappa^{*}=0.01 \text {. }
$$

Figure 6 shows that the gas bubble microstructure snapshots at $t^{*}=12000$ for different mobility of Xe at the GB with $\kappa^{*}=0.01$. We can see a tendency that the grain face gas bubbles grow faster and have elongated morphology with increasing mobility at the GB. As a consequence, a gas tunnel at the GB will form faster. Figure 7 displays the temporal gas bubble evolution in the whole simulation cell when $\Delta E_{X e}^{m} / E_{X e}^{m 0}=0, \kappa^{*}=0.01$ and a different initial gas bubble distribution from Fig. 6(a). As we expected, there is no obvious difference of gas bubble coarsening kinetics between inside the grain and near the grain boundaries because of the small simulation cell. But it clearly shows the evolution of the grain boundary and grain face gas bubble shape to satisfy the equilibrium among grain boundary tension and gas bubble interface tensions. Again, the GB migration can be observed. 


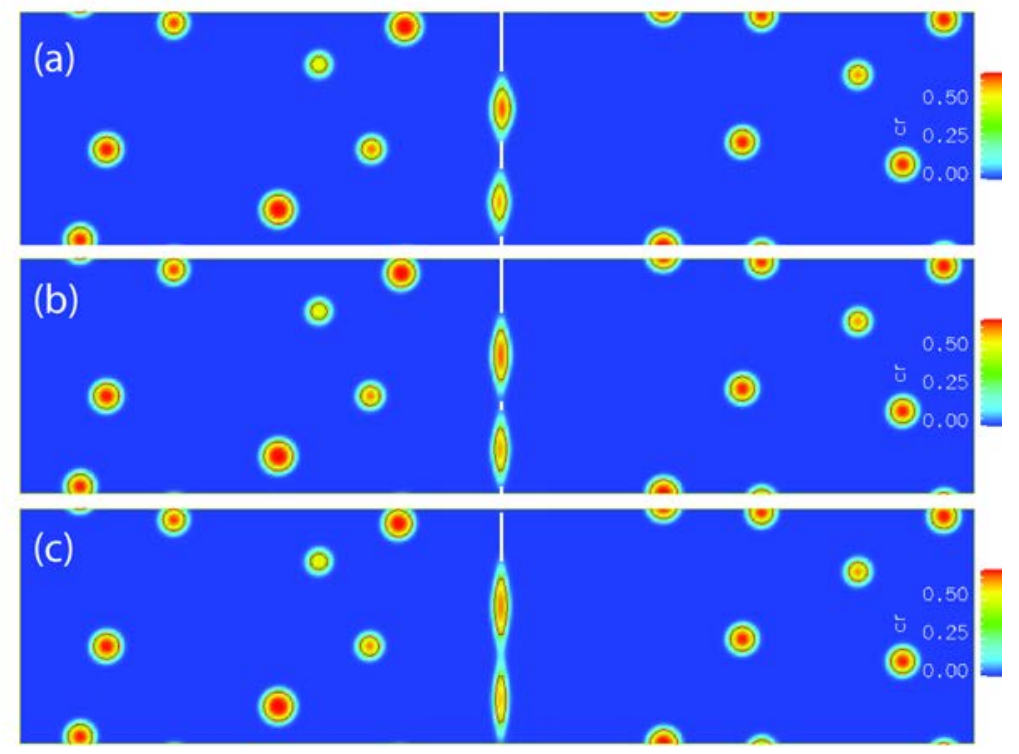

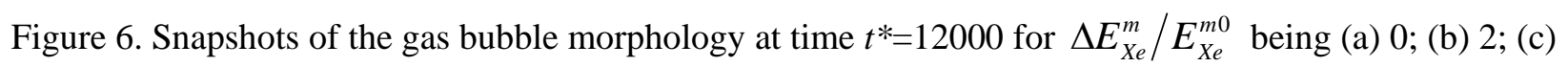
4. 


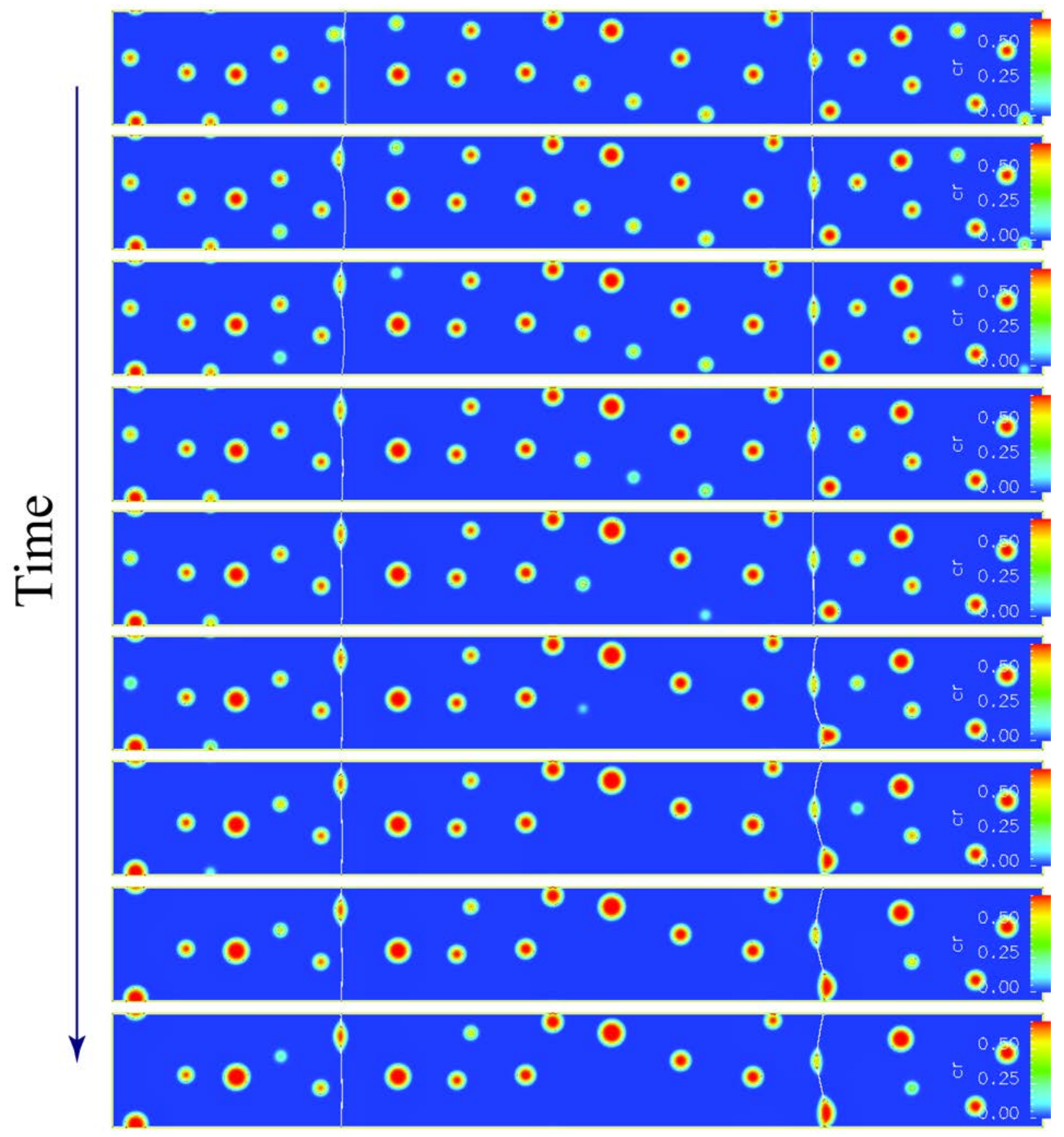

Figure 7. Snapshots of gas bubble temporal evolution from $t^{*}=0$ to $t^{*}=12000$ when $\Delta E_{X e}^{m} / E_{X e}^{m 0}=0$,

$$
\kappa^{*}=0.01
$$




\section{CONCLUSIONS}

We have enhanced the generic PF model by enabling it to simulate the effect of thermodynamic and kinetic properties of the system on gas bubble microstructure evolution kinetics and morphology during post-irradiation thermal annealing in polycrystalline $\mathrm{UO}_{2}$ materials. Preliminary simulations demonstrated that the model reasonably predicts GB segregation, grain face gas bubble morphology, the effect of mobility inhomogeneity on gas bubble growth kinetics. However, for quantitative simulations, we need 1) more validation of the model, especially the relation between model parameters and interface properties; 2) accurate atomistic simulation results on GB properties such as atomic structures, formation energy, and migration energy to develop the functional used in our model; and 3) benchmark the gas bubble evolution kinetics against experiments.

\section{ACKNOWLEDGMENTS}

This work was supported by the U.S. Department of Energy’s Nuclear Energy Advanced Modeling and Simulation (NEAMS) Program at the Pacific Northwest National Laboratory, which is operated by Battelle for the U.S. Department of Energy under Contract No. DE-AC05-76RL01830.

\section{REFERENCES}

[1] Y.L. Li, S.Y. Hu, R. Montgomery, F. Gao, X. Sun, M. Tonks, B. Biner, P. Millett, V. Tikare, B. Radhakrishnan, D. Andersson, Mesoscale Benchmark Demonstration Problem 1: Mesoscale Simulations of Intra-granular Fission Gas Bubbles in $\mathrm{UO}_{2}$ under Post-irradiation Thermal Annealing, DOE-FCR\&DMDSM-2012-000098 (2012).

[2] M.E. Cunningham, M.D. Freshley, D.D. Lanning, J Nucl Mater 200 (1993) 24.

[3] S. Kashibe, K. Une, K. Nogita, J Nucl Mater 206 (1993) 22.

[4] R.J. White, The growth of intra-granular bubbles in post-irradiation annealed UO2 fuel, in: IAEA Technical committee on nuclear fuel behaviour modeling at high burn-up and its experimental support, Lake Windermere, UK, 2000.

[5] D.R. Olander, D. Wongsawaeng, J Nucl Mater 354 (2006) 94.

[6] C. Baker, J Nucl Mater 71 (1977) 117.

[7] J.M. Griesmeyer, N.M. Ghoniem, J Nucl Mater 80 (1979) 88.

[8] D.A. Macinnes, I.R. Brearley, J Nucl Mater 107 (1982) 123.

[9] P. Losonen, J Nucl Mater 304 (2002) 29.

[10] P. Losonen, J Nucl Mater 280 (2000) 56.

[11] C.C. Dollins, F.A. Nichols, J Nucl Mater 66 (1977) 143.

[12] J. Rest, S.A. Zawadzki, FASTGRASS: A Mechanistic Model for the Prediction of Xe, I, Cs, Te, Ba and Sr Release from Nuclear Fuel under Normal and Severe-Accident Conditions, NUREG/CR-5840 TI92 040783 (1994). 
[13] T.J. Heame, D.A. Williams, N.E. Bixler, A.J. Grimley, C.J. Wheatley, N.A. Johns, P.Domagala, L.W. Dickson, C.A. Alexander, I. Osborn-Lee, S. Zawadzki, J. Rest, A. Mason, R.Y. Lee, VICTORIA: A Mechanistic Model of Radionuclide Behavior in the Reactor Coolant System under Severe Accident Conditions, NUREG/CR-5545 (1992).

[14] M.S. Veshchunov, V.D. Ozrin, V.E. Shestak, V.I. Tarasov, R. Dubourg, G. Nicaise, Nucl. Eng. Des. 236 (2006) 179.

[15] M.S. Veshchunov, R. Dubourg, V.D. Ozrin, V.E. Shestak, V.I. Tarasov, J Nucl Mater 362 (2007) 327.

[16] G.A. Berna, C.E. Beyer, K.L. Davis, D.D. Lanning, FRAPCON-3: A Computer Code for the Calculation of Steady-State, Thermal Mechanical Behavior of Oxide Fuel Rods for High Burnup, NUREG/CR-6534, vol. 2 (PNNL-11513 v.2, Pacific Northwest National Laboratory, Richland, WA) (1997).

[17] A. Karma, W.J. Rappel, Phys Rev E 57 (1998) 4323.

[18] Y.M. Jin, A. Artemev, A.G. Khachaturyan, Acta Materialia 49 (2001) 2309.

[19] L.Q. Chen, Ann. Rev. Mater. Res. 32 (2002) 113.

[20] S.Y. Hu, C.H. Henager, L.Q. Chen, Acta Mater 58 (2010) 6554.

[21] Y.L. Li, S.Y. Hu, Z.K. Liu, L.Q. Chen, Acta Mater 50 (2002) 395.

[22] I. Steinbach, Model. Simul. Mater. Sci. Eng. 17 (2009).

[23] S.Y. Hu, C.H. Henager, Acta Mater 58 (2010) 3230.

[24] I. Zacharie, S. Lansiart, P. Combette, M. Trotabas, M. Coster, M. Groos, J Nucl Mater 255 (1998) 92.

[25] S.G. Kim, W.T. Kim, T. Suzuki, Phys Rev E 60 (1999) 7186.

[26] Y.L. Li, S.Y. Hu, X. Sun, F. Gao, C.H. Henager, M. Khaleel, J Nucl Mater 407 (2010) 119.

[27] C. Ronchi, J Nucl Mater 96 (1981) 314.

[28] S.Y. Hu, L.Q. Chen, Acta Materialia 49 (2001) 1879.

[29] J.B. Wachtman, M.L. Wheat, H.J. Anderson, J.L. Bates, J Nucl Mater 16 (1965) 39.

[30] J.W. Cahn, Acta Metall. 9 (1961) 795.

[31] J.W. Cahn, S.M. Allen, Le J. de Phys. Col. 38 (1977) 51.

[32] J.W. Cahn, J.E. Hilliard, J. Chem. Phys. 28 (1958) 258.

[33] L.Q. Chen, J. Shen, Comput. Phys. Commun. 108 (1998) 147.

[34] K. Nogita, K. Une, Nuclear Instruments \& Methods in Physics Research Section B-Beam Interactions with Materials and Atoms 91 (1994) 301. 


\section{APPENDIX A: THERMODYNAMIC AND KINETIC PROPERTIES OF GRAIN BOUNDARIES}

\section{Grain boundary energies, atomic structure and stresses near GBs}

The experimental data indicated that $\sim 16 \%$ of the boundaries were CSL (coincident site lattice) boundaries and the CSL distribution was dominated by low $\sum$ boundaries; namely $\sum 9, \sum 3$, and $\sum 5$ [1]. Based on these experimental observations, two representative GBs (GBs), $\sum 3$ and $\Sigma 5$, will be considered in the present work. Starting from general-twin slab configurations, $\gamma$ surfaces were calculated to search for the minimum energy structures of the GBs. We used the interatomic potential developed by Yakub et al. [2] to describe the interactions of $\mathrm{UO}_{2}$. The stable GBs are shown in Fig. 1.

After constructing the minimum energy structures of the two different GBs, the GB energy will be calculated using the following equation [1]

$$
E_{G B}=\frac{E_{G B}^{t o t}-n E_{b u l k}}{2 A},
$$

where $E_{G B}^{t o t}$ is the total energy of the simulation box including the GB, $E_{\text {bulk }}$ is the total of bulk $\mathrm{UO}_{2}$ per unit, $n$ is the total number of $\mathrm{UO}_{2}$ units in the box, and $A$ is the area of the GB interface. Our results will be compared to those in Ref. [1].

The stress of $\sum 3$ and $\sum 5$ GBs will be calculated using molecular statics, which is similar to that of $\sum 3$ GB in iron, as shown in Fig. 2. From Fig. 2, it is clearly seen that the stress is distributed within about 1nm near the interface, which suggests that the noble gas may be trapped in the regions of high stress concentration.

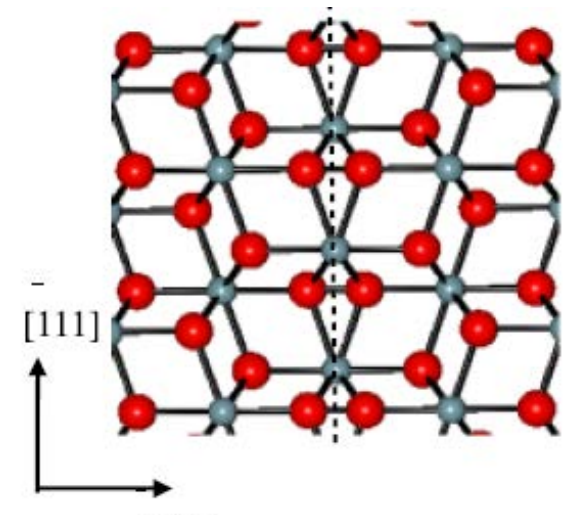

[110]

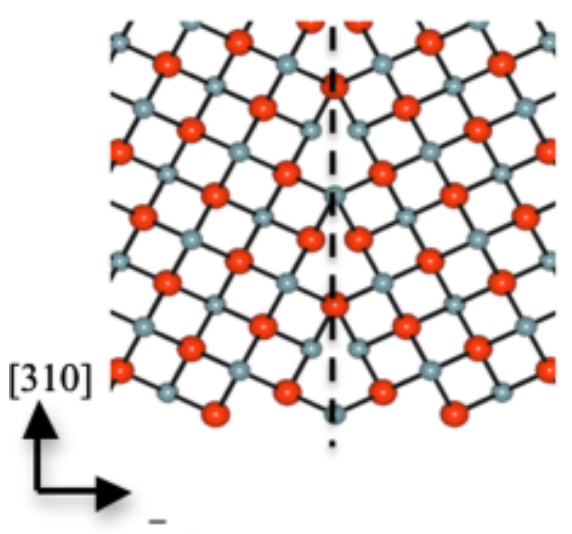

[130]

Fig. 1. Structure of GBs in $\mathrm{UO}_{2}$ : (a) $\sum 3$ and (b) $\sum 5$. Small and big spheres represent $\mathrm{U}$ ions and $\mathrm{O}$ ions, respectively. 


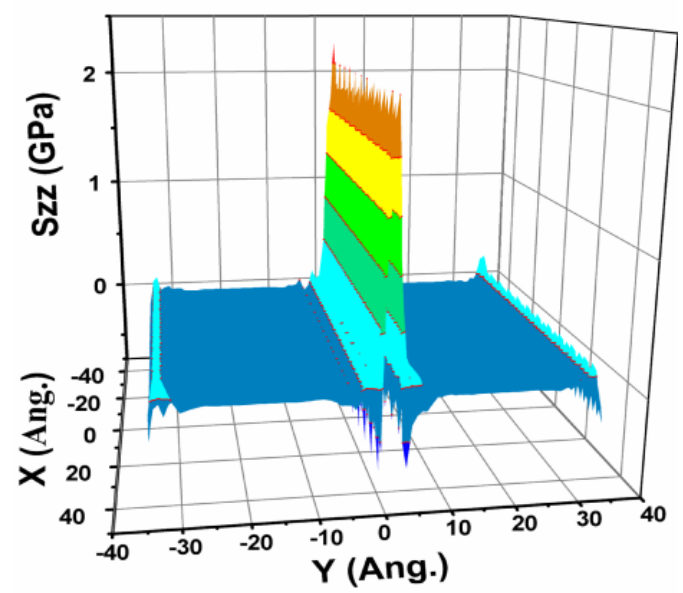

Fig. 2. Stress distribution on the XOY plane of $\Sigma 3\{112\}$ in $\alpha$-Fe.

\section{Properties of Xe-vacancy $\left(X e_{n} \underline{V}_{\underline{m}}\right)$ clusters around $\mathrm{GBs}$ in $\mathrm{UO}_{2}$}

According to the stress distribution of $\mathrm{GBs}$ in $\mathrm{UO}_{2}$, the Xe-vacancy clusters will be placed in the region of high stress concentration. The fate of $\mathrm{Xe}_{\mathrm{n}} \mathrm{V}_{\mathrm{m}}$ clusters in and around the interface of $\mathrm{GBs}_{\mathrm{in}} \mathrm{UO}_{2}$ will be investigated using molecular statics, molecular dynamics and the dimer method. Firstly, within about $1 \mathrm{~nm}$ from the interface, $\mathrm{Xe}_{\mathrm{n}} \mathrm{V}_{\mathrm{m}}$ clusters are initially placed in different distance from the interface and relaxed to minimum energy configurations using molecular statics. The formation energies of $\mathrm{Xe}_{\mathrm{n}} \mathrm{V}_{\mathrm{m}}$ clusters around the GB will be studied using following equations:

$$
E_{X e_{n} V_{m}}^{f}=E_{G B+X e_{n} V_{m}}^{t o t}-E_{G B}^{t o t}+m \times E^{d}-n \times E^{X e},
$$

where $E_{G B+X e_{n} V_{m}}^{t o t}$ and $E_{G B}^{t o t}$ are the total energy of the crystal which includes the GB, with and without the $\mathrm{Xe}_{\mathrm{n}} \mathrm{V}_{\mathrm{m}}$ cluster, respectively. $E^{d}$ is the cohesive energy of $\mathrm{U}$ or $\mathrm{O}$ ion, $E^{X e}$ is the cohesive energy of fcc Xe. Especially, for $\mathrm{n}=0$ and $\mathrm{m}=1, E_{X e_{n} V_{m}}^{f}$ becomes to $E_{v a c}^{f}$, which is the formation energy of a vacancy (It should be noted that the vacancy may be an oxygen vacancy or an uranium vacancy.); for $n=1$ and $\mathrm{m}=0, E_{X e_{n} V_{m}}^{f}$ is the formation energy of an interstitial Xe atom $\left(E_{X e}^{f}\right)$, from which the binding energy of a Xe atom to the GBs can be determined; for $\mathrm{n}=1$ and $\mathrm{m}=1, E_{\mathrm{Xe}_{n} V_{m}}^{f}$ indicates the formation energy of a substitutional Xe ( $E_{\text {sub }}^{f}$ ) in the GBs. Because of two kinds of vacancies in $\mathrm{UO}_{2}$, the substitutional Xe may sites at the $\mathrm{U}$ or $\mathrm{O}$ lattice position. The formation energies of $\mathrm{Xe}_{\mathrm{n}} \mathrm{V}_{\mathrm{m}}$ clusters as a function of distance to the GB interface will be studied in details.

The binding properties of the $\mathrm{Xe}_{\mathrm{n}} \mathrm{V}_{\mathrm{m}}$ clusters to GBs in $\mathrm{UO}_{2}$ will be also studied as a function of distance to the GB plane. The binding energies of the $\mathrm{Xe}_{\mathrm{n}} \mathrm{V}_{\mathrm{m}}$ clusters to GBs are obtained from the equation [3]:

$$
E_{X e_{n} V_{m}}^{b}=E_{X e_{n} V_{m}}^{t o t}-E_{G B}^{t o t}-E_{b u l k, X e_{n} V_{m}}^{f},
$$


where $E_{G B+X e_{n} V_{m}}^{t o t}$ and $E_{G B}^{t o t}$ are the same as those in Eq. (A-2), $E_{b u l k, X e_{n} V_{m}}^{f}$ is the formation energy of a $\mathrm{Xe}_{\mathrm{n}} \mathrm{V}_{\mathrm{m}}$ cluster in bulk $\mathrm{UO}_{2}$. The various binding energies in the different GBs will affect the diffusion and migration mechanisms of $\mathrm{Xe}$ atoms in $\mathrm{UO}_{2}$.

\section{Dynamic behavior of Xe atoms and small Xe clusters at GBs in $\mathrm{UO}_{2}$}

Firstly, the migration mechanisms of self-interstitials, vacancies and Xe interstitial at the $\sum 3$ and $\sum 5$ GBs are investigated using dimmer method. The results are compared to those in bulk.Then, the diffusion properties of Xe interstitials and Xe clusters in $\sum 3$ and $\sum 5$ GBs at different temperatures will be obtained using molecular dynamics simulations. The positions $\mathrm{r}_{i}(t)$ of all the atoms at time $t$ are recorded and the mean-square displacement (MSD) is determined by [3]

$$
M S D=<\left|r_{i}(t)-r_{i}(0)\right|^{2}>=\frac{1}{N} \sum_{i=1}^{N}\left|r_{i}(t)-r_{i}(0)\right|^{2},
$$

where $N$ is the total atomic number and ' $<\cdots>$ ' denotes averaging over all the atoms. The diffusion coefficient $(D)$ can be obtained with the Einstein-Smoluchowski relation:

$$
D=\lim _{t \rightarrow \infty} \frac{M S D}{q_{i} t}=\lim _{t \rightarrow \infty} \frac{1}{q_{i} t}<\left|r_{i}(t)-r(0)\right|^{2}>
$$

where $q_{i}$ is a numerical constant that depends on the dimensionality such that $q_{i}=2,4$, or 6 for the one-, two- or three-dimensional diffusion, respectively. The relation of the migration energy $\left(E_{m}\right)$ to the diffusion coefficient $D$ is based on the Arrhenius equation:

$$
D=D_{0} \exp \left(-E_{m} / k_{B} T\right) .
$$

The $E_{m}$ and $D$ can be obtained by fitting the diffusion coefficient to equation (A-6) over a range of temperatures.

\section{References:}

[1] P. V. Nerikar, K. Rudman, T. G. Desai, D. Byler, C. Unal, K. J. McClellan, S. R. Phillpot, S.B. Sinnott, P. Peralta, B. P. Uberuaga and C.R. Stanek, J. Am. Ceram. Soc., 94 (2011) 1893-1900.

[2] E. Yakub, C. Ronchi, D. Staicu, J. Chem. Phys., 127 (2007) 094508.

[3] F. Gao, H. Heinisch, R.J. Kurtz, J Nucl. Mater. 351 (2006) 133-140. 


\section{APPENDIX B: INITIAL CONDITION AND MATERIAL PROPERTIES USED IN SIMULATIONS.}

Table B - 1. Initial condition. [1]

\begin{tabular}{l|l}
\hline Description & Value \\
\hline $\begin{array}{l}\text { Initial bubble density } \\
\text { Initial gas concentration in } \\
\text { matrix }\end{array}$ & $9 \times 10^{23} / \mathrm{m}^{3}$ \\
$\begin{array}{l}\text { Initial U/O vacancy } \\
\text { concentration in matrix }\end{array}$ & 0.0042 \\
$\begin{array}{l}\text { Dislocation density and types } \\
\text { Initial gas atom concentration }\end{array}$ & $2 \times 10^{14} / \mathrm{m}^{3}$ \\
in bubbles & 0.7 \\
Initial bubble distribution & Normal distribution of radius \\
Mean bubble radius & $1 \mathrm{~nm}$ \\
\hline
\end{tabular}

Table B - 2. Thermodynamic and kinetic properties of defects in $\mathrm{UO}_{2}$. [1]

\begin{tabular}{l|l|}
\hline Description & Value \\
\hline Xe migration energy in $\mathrm{UO}_{2}$ & $3.9,4.5 \mathrm{eV}$ \\
$\mathrm{U}_{\mathrm{V}} / \mathrm{O}_{\mathrm{V}} /$ Xe complex migration energy & $3.9,4.5 \mathrm{eV}$ \\
Xe formation energy & $1.8 \sim 3.0 \mathrm{eV}$ \\
$\mathrm{U}$ vacancy formation energy & $1.8 \sim 3.0 \mathrm{eV}$ \\
Formation volume of $\mathrm{U}$ vacancy & $42.3\left[\AA^{3}\right]$ \\
Formation volume of Xe & $50.15\left[\AA^{3}\right]$ \\
Interfacial energy of gas bubbles & $0.6 \mathrm{~J} / \mathrm{m}^{2}$ \\
Critical gas bubble size & $0.8 \mathrm{~nm}$ \\
Elastic constants $\mathrm{C}_{11}, \mathrm{C}_{12}$ and $\mathrm{C}_{44}$ of $\mathrm{UO}_{2}$ & $395 \mathrm{GPa}, 121 \mathrm{GPa}, 64 \mathrm{GPa}$ \\
\hline
\end{tabular}

[1] Y.L. Li, S.Y. Hu, R. Montgomery, F. Gao, X. Sun, M. Tonks, B. Biner, P. Millett, V. Tikare, B. Radhakrishnan, D. Andersson, Mesoscale Benchmark Demonstration Problem 1: Mesoscale Simulations of Intra-granular Fission Gas Bubbles in $\mathrm{UO}_{2}$ under Post-irradiation Thermal Annealing, DOE-FCR\&DMDSM-2012-000098 (2012). 